Discussion/conclusion Despite a robust and clear guideline on epididymo-orchitis our results show that antibiotic prescribing is often incorrect. Furthermore, the work-up for an STI as a cause of epididymo-orchitis is incomplete.

\section{P13 WHAT TO DO IN A SYPHILIS OUTBREAK}

Louise Seppings*, Alan Tang, Fabian Chen. Royal Berkshire Hospital, Reading, UK

\subsection{6/sextrans-2015-052126.57}

Background/introduction In Autumn 2014 a surprising number of patients were being diagnosed with early syphilis, in the sexual health clinic, Reading. From January 2014 to January 2015 twenty-one early syphilis cases arose. Whereas 2013 totalled 5 cases, which was an average year.

Aims/objectives To identify if this constituted an outbreak. Determine why increasing numbers of early syphilis were arising and which patients groups were at risk. To prevent further cases. Methods January to September cases were reviewed retrospectively and then new cases prospectively. Public Health England was notified and an action meeting ensued. Patient behaviours and contact tracing data collected. Letters written to inform healthcare services. Clinic information boards and website updated. Social media and appropriate charity organisations approached to reach target groups.

Results Eight presented with primary syphilis, ten with secondary and three with early latent. Eighteen cases were men who have sex with men (MSM), highlighting the main at risk group. Seven of the MSM were HIV positive with three being newly diagnosed. The average number of sexual contacts was twelve with one third using social networking apps to meet.

Discussions/conclusions Syphilis outbreak confirmed. MSM patients are the main risk group with one third HIV co-infection, which is a concern. Common usage of social networking apps identified to meet sexual partners, which can involve serosorting. Collaboration between sexual and Public Health teams resulted in raising awareness. Hopefully these measures will reduce the number of cases but it will require close monitoring.

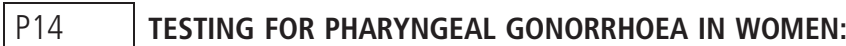 AN IMPORTANT RESERVOIR OF INFECTION, OR EXCESSIVE FALSE POSITIVE DIAGNOSES}

Georgina Forbes, Rachel Drayton*. Cardiff and Vale University Health Board, Cardiff, UK

10.1136/sextrans-2015-052126.58

Background In 2012 we reported that 30\% of heterosexual women attending our service had a positive gonorrhoea (GC) NAAT on pharyngeal sampling, without infection elsewhere. A PPV of $87 \%$ has been reported for our pharyngeal samples, but confirmatory GC NAATs remain routinely not available locally. Due to concerns about false positives, we subsequently restricted pharyngeal testing to women at higher risk of infection at this site only and reviewed the findings.

Methods All positive GC NAATs in women attending our service from October 2013 to March 2014 were reviewed. Findings were compared to the data from January to July 2012. All NAATs were performed on Roche Cobas 4800.

Results There were 36 women in the 2014 sample, compared to 40 in the 2012 sample. Of these, 19 (53\%) had a positive GC NAAT on a pharyngeal sample, compared to $17(43 \%)$ in the
2012 sample $(\mathrm{p}=0.38) .13(36 \%)$ of women with a positive GC NAAT had the infection detected on pharyngeal swab only in the 2014 sample, compared to $12(30 \%)$ in the 2012 sample $(\mathrm{p}=0.56)$.

Discussion By restricting testing to women at higher risk of pharyngeal only infection, we found 36\% women had an isolated positive pharyngeal GC NAAT, and would not have been diagnosed if pharyngeal sampling was not taken. Further work is needed assessing the performance of the Roche Cobas 4800 in this population in order to evaluate the proportion of false positive diagnoses versus the extent of this potential reservoir of infection.

\section{P15 AORTITIS REQUIRING CARDIOTHORACIC SURGERY IN A CASE OF SECONDARY SYPHILIS}

Pippa Newton, Cara Saxon, Sameena Ahmad*. University Hospital South Manchester, Manchester, UK

\subsection{6/sextrans-2015-052126.59}

Background/introduction Cardiac complications of syphilis typically occur 10-30 years after being infected. There has been a recent case of aortitis in secondary syphilis in the literature.

Aim(s)/objectives To report a case of syphilitic aortitis in a patient recently infected with syphilis.

Methods Case report.

Results A 37-year-old white British female was found wandering the streets semi-clothed by paramedics. Background: bipolar/schizoaffective disorder with previous psychosis and known substance misuse. A loud early diastolic murmur was found on examination. An ECG revealed anterior T wave changes. Troponin was $>2000 \mathrm{ng} / \mathrm{L}$ and echocardiogram $(\mathrm{ECHO})$ revealed a dilated left ventricle with severe aortic regurgitation (AR). Transoesophageal ECHO demonstrated an oedematous, thickened aortic root. CT aortogram confirmed aortitis. Syphilis serology was positive (RPR 1:256). She had a male partner of 5 years and had never had a syphilis test before. Due to penicillin allergy she was commenced on Doxycycline for 28 days with adjuvent. Three weeks into treatment she developed heart failure and was admitted to intensive care. ECHO revealed an ejection fraction of $30 \%$ and progressive valvular pathology. Following desensitisation she commenced on benzylpenicillin plus probenacid for 17 days. Two weeks into treatment she underwent an aortic valve replacement and coronary artery bypass graft (x2). After a protracted recovery she was discharged two months later and remains under cardiology follow up.

Discussion/conclusion Whilst it is not exactly clear when this patient acquired syphilis the high RPR titres suggest that infection was recent. This case demonstrates a rare but serious and life-threatening complication of early syphilis.

\section{P16 LGV-AN INNER CITY COHORT}

Priyanka Saigal*, Mannampallil (Itty) Samuel, Manpreet Bahra, Michael Brady, Chris Taylor. King's College Hospital, London, UK

\section{$10.1136 /$ sextrans-2015-052126.60}

Background/introduction LGV is hyperendemic amongst MSM in the UK. There is a strong association with HIV and hepatitis $\mathrm{C}$ infections. 